\title{
Association of Trimethylamine-N-Oxide Levels with Risk of Cardiovascular Disease and Mortality among Elderly Subjects: A Systematic Review and Meta-Analysis
}

\author{
Guixiu Chen Lin He Xiaotao Dou Tao Liu
}

Department of Cardiovascular Medicine, The Second Clinical Medical College of North Sichuan Medical College, Nanchong Central Hospital, Nanchong, China

\section{Keywords}

Trimethylamine-N-oxide · Adverse cardiovascular events .

All-cause mortality · Meta-analysis · Elderly

\begin{abstract}
Background: Trimethylamine-N-Oxide (TMAO) is a microbiome-related metabolite that has been linked to cardiovascular and renal function. This study has reviewed and analyzed the relationship between TMAO and all-cause mortality and adverse cardiovascular events in elderly subjects. Methods: We determined whether this association was modified in the presence of chronic kidney disease (CKD), heart failure (HF), and diabetes. Based on the criteria, systematic review and meta-analysis were conducted and performed. Results: A total of 27 prospective cohort studies were retrieved finally to examine the associations. The high TMAO was positively associated with all-cause mortality (hazard ratio (HR): 1.38 [95\% confidence interval (Cl): 1.306-1.460]) as well as adverse cardiovascular events (HR: 1.032 [95\% Cl: 1.014-1.051]). The association remained upon subgroup analysis for patients with CKD and HF but no association found for the patients with diabetes (HR: 1.15 [95\% Cl, 0.81-1.64]). Conclu-
\end{abstract}

Karger@karger.com www.karger.com/crm

Karger $\stackrel{\text { ' }}{5}$

GOPEN ACCESS
(C) 2021 The Author(s)

Published by S. Karger AG, Basel

This is an Open Access article licensed under the Creative Commons Attribution-NonCommercial-4.0 International License (CC BY-NC) (http://www.karger.com/Services/OpenAccessLicense), applicable to the online version of the article only. Usage and distribution for commercial purposes requires written permission. sions: The findings of this review revealed that the TMAO level is associated with all-cause mortality and adverse cardiovascular events.

(c) 2021 The Author(s).

Published by S. Karger AG, Basel

\section{Introduction}

Cardiovascular disease (CVD) is the leading cause of mortality worldwide, attributed to more than $30 \%$ of annual global mortality [1]. Recent research directs at identification of novel prognostic biomarkers for the detection of cardiovascular risk. The trimethylamine- $\mathrm{N}$-oxide (TMAO) is a gut microbiome-generated compound whose blood concentration increases after ingesting dietary L-carnitine and phosphatidylcholine rich foods. It has gained recent interest due to its demonstrated effects on atherosclerosis, defects in cholesterol metabolic pathways, and promoting endothelial dysfunction; thereby, affecting lipid metabolism and inflammatory response in animal models. All of these phenomena underlie CVD in humans. Furthermore, several reports show that TMAO may predict (recurrent) $\mathrm{CV}$ events in high CV risk popu- 


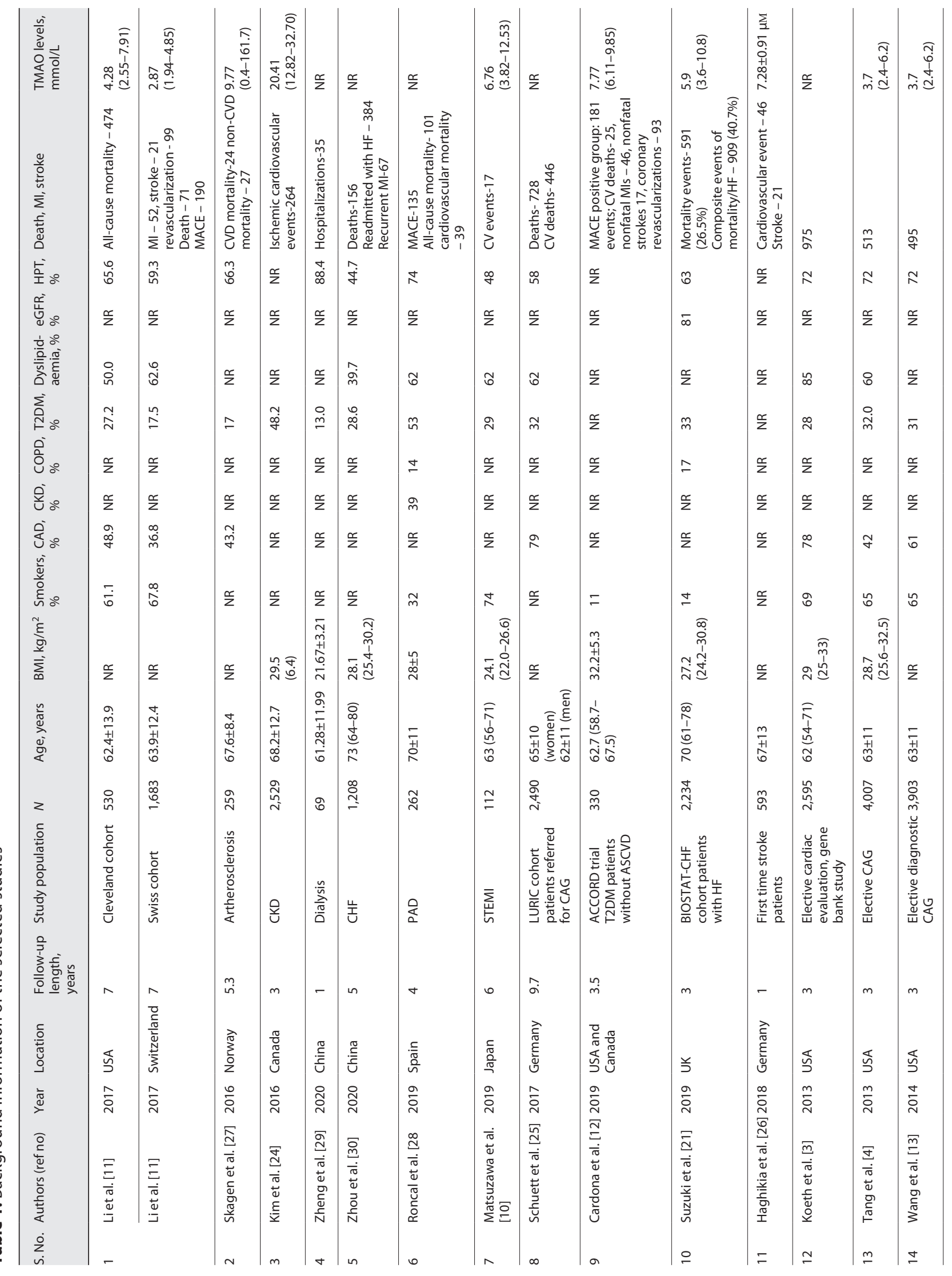




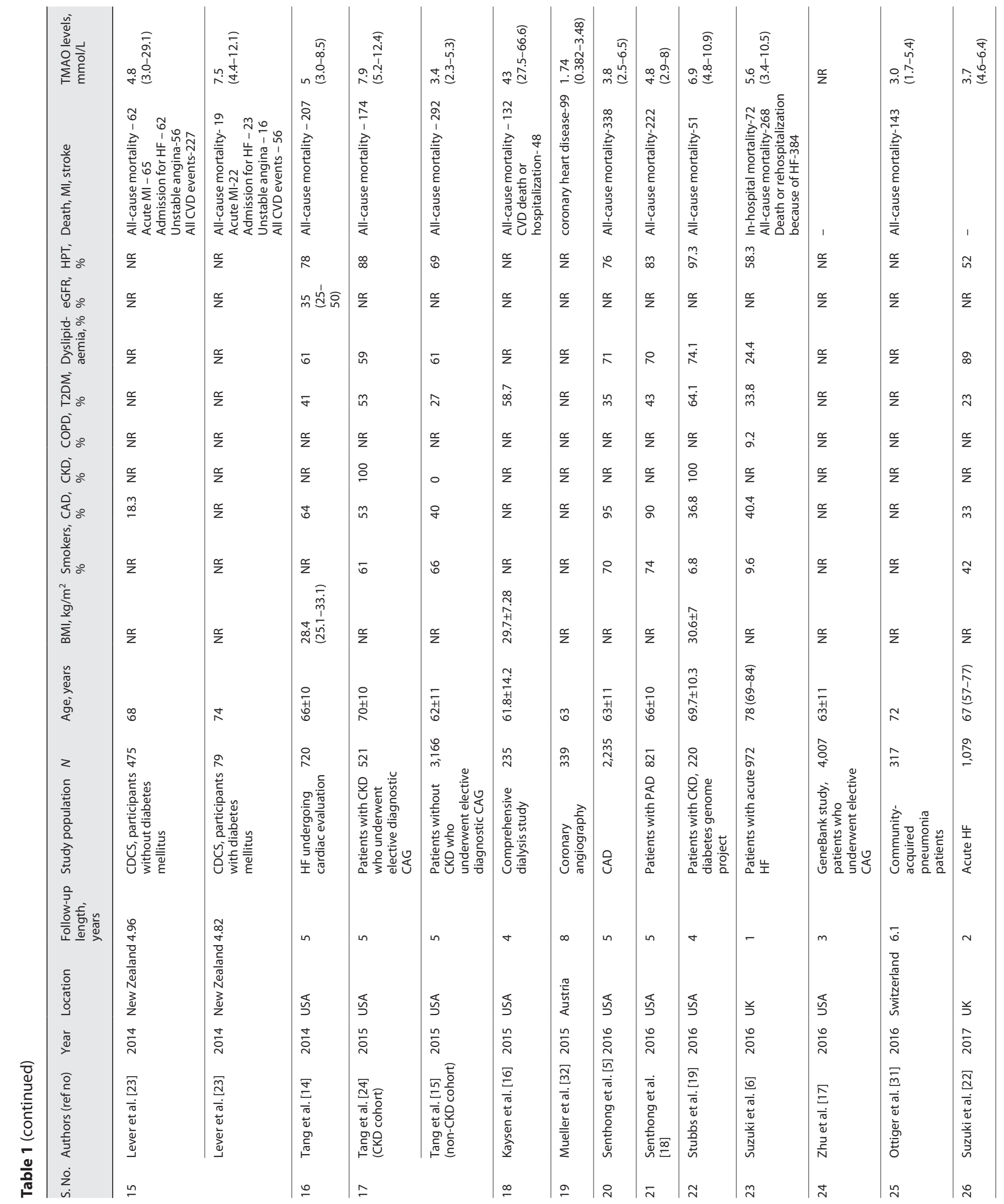



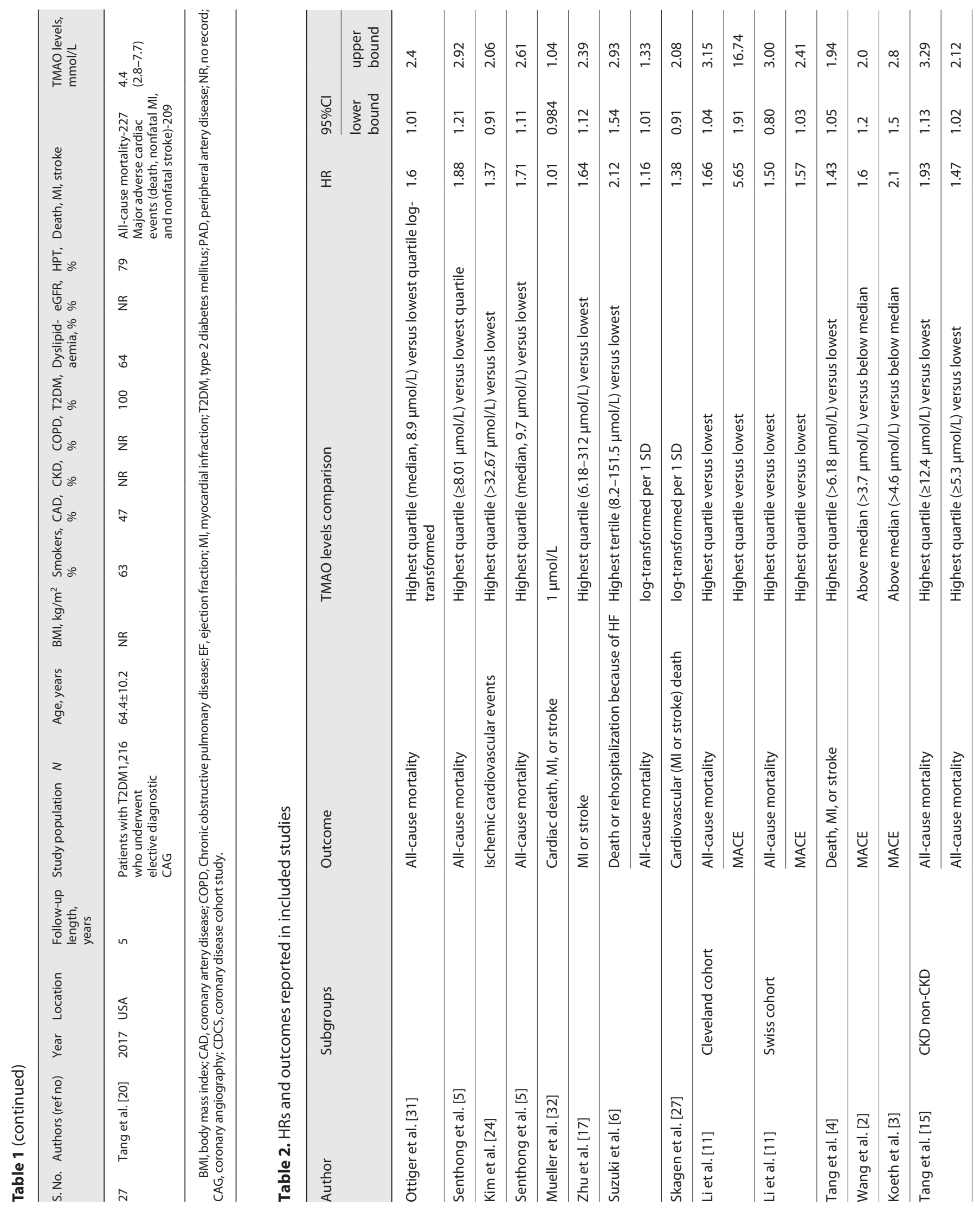


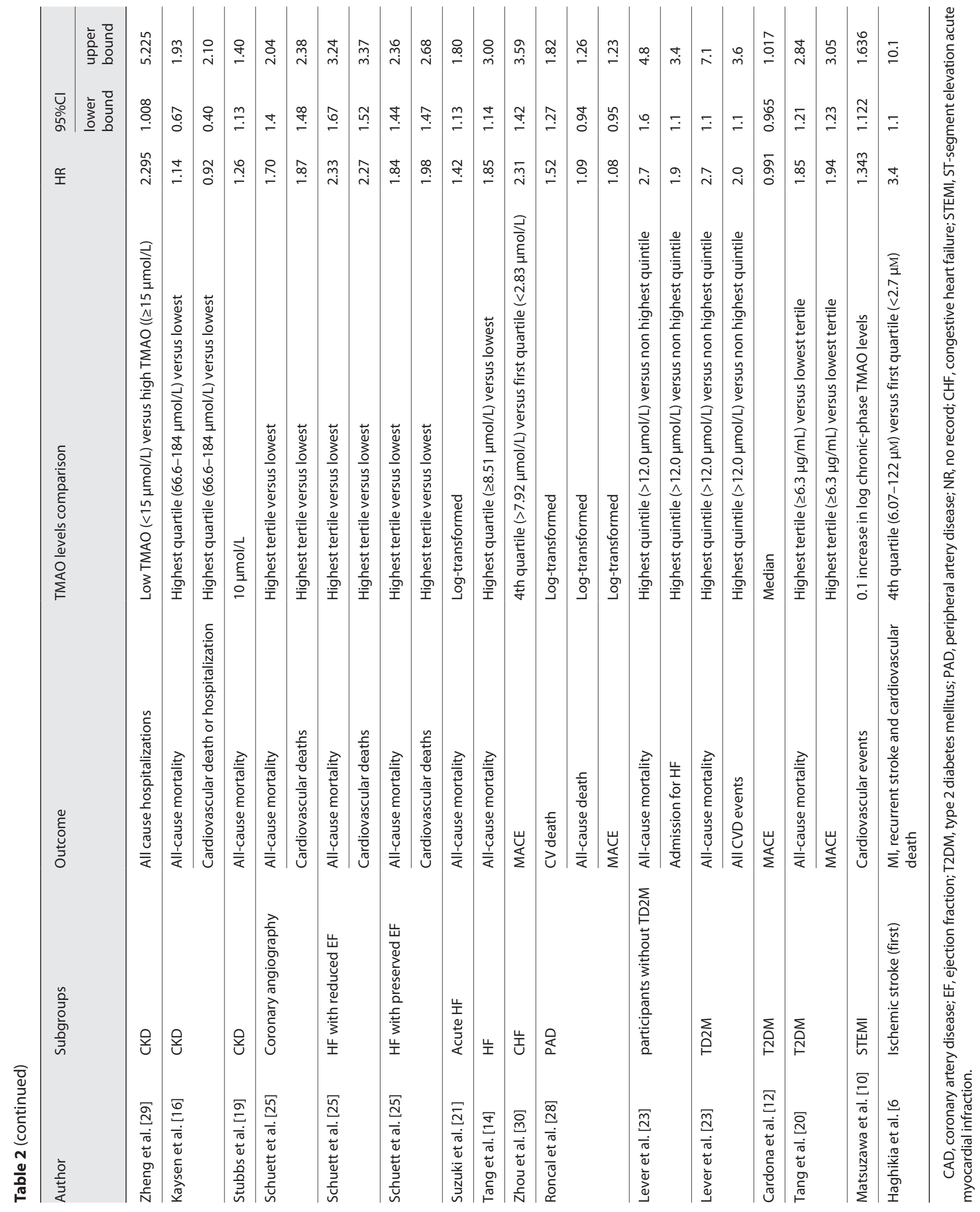


Fig. 1. PRISMA flowchart of the selected studies. PRISMA, Preferred Reporting Items for Systematic Reviews and MetaAnalyses.

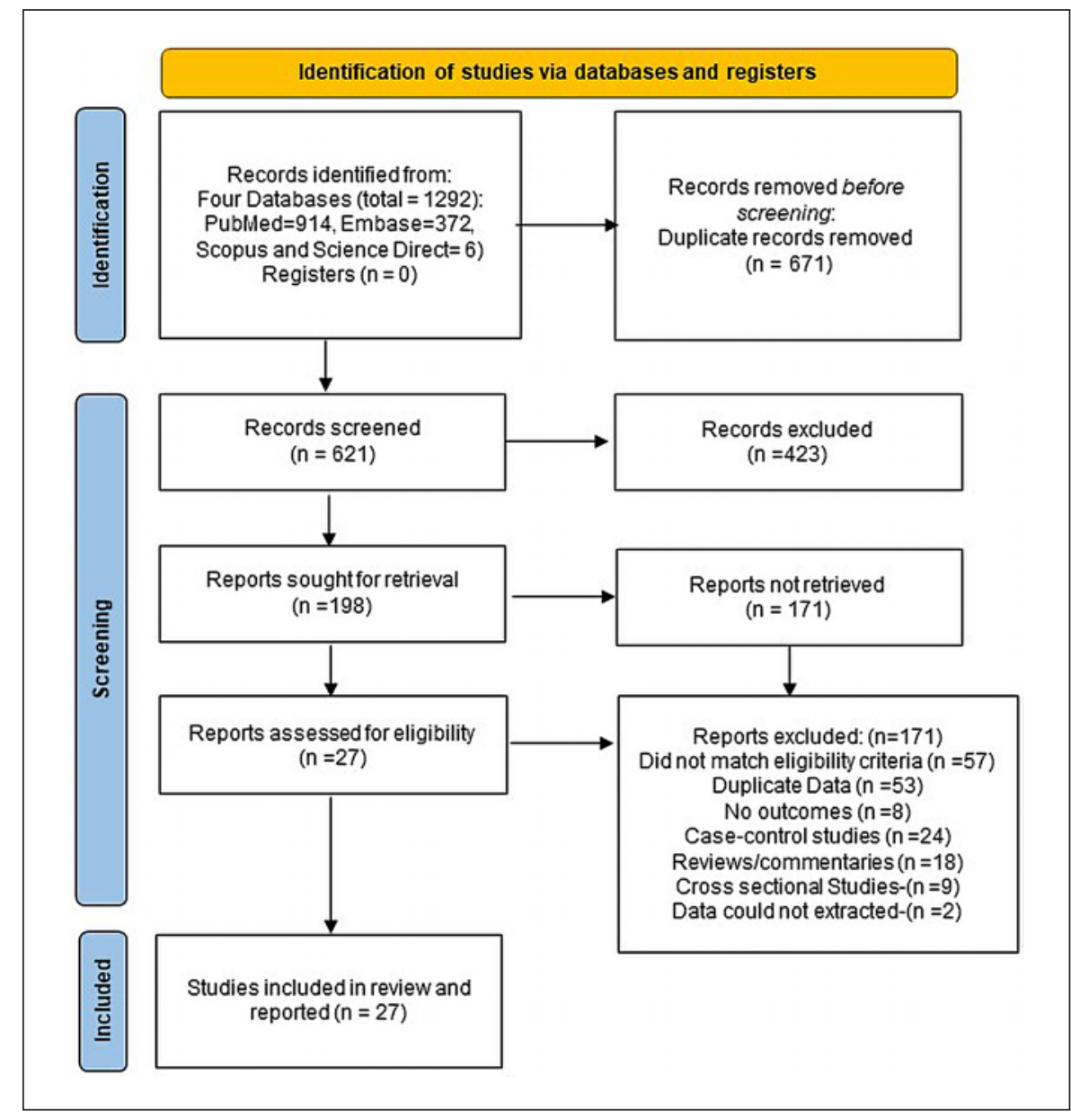

lations [2-6]. In a recent meta-analysis [7], the authors reported that participants having high TMAO had an increased hazard ratio (HR) of mortality by $47 \%$; thereby suggesting some role of TMAO in all-cause mortality.

The elderly population is increasing globally [8]. However, the quality of life is compromised often due to longevity affected by residual complications of chronic disorders, notably heart diseases and their sequelae. Other common diseases that they suffer from are chronic kidney disease and diabetes, leading to repeated cardiovascular events and premature death [9]. The TMAO levels studied across all age-groups has been associated with future risk of adverse events, but whether they present increased risk or not among the older age-group is unknown. Some studies have been inconclusive to determine the association of TMAO with mortality and cardiovascular risks. For instance, one recent study reported no significant correlation between acute-phase TMAO level and the incidence of major adverse cardiovascular events (MACE) [10].
Therefore, in this study, we have designed to address these previous gaps and performed a systematic review and meta-analysis on observational studies to quantitatively evaluate the association between baseline circulating TMAO and all-cause mortality and the risk of subsequent cardiovascular events in the elderly age-group. In addition, we further analyzed subgroups of chronic kidney disease, heart failure (HF), and diabetes to identify if the excess risk was associated with these conditions.

\section{Material and Methods}

\section{Literature Search Strategy}

The present study was designed according to Preferred Reporting Items for Systematic Reviews and Meta-Analyses guidelines and the Meta-analysis of Observational Studies in Epidemiology (MOOSE) guidelines. The complete Preferred Reporting Items for Systematic Reviews and Meta-Analyses checklist is provided in Table 1. Articles were retrieved from the four databases: Pubmed, Embase, SCOPUS, and ScienceDirect for studies on TMAO from inception until December 2020. Studies were identified using the
44

Cardiorenal Med 2022;12:39-54 DOI: $10.1159 / 000520910$
Chen/He/Dou/Liu 


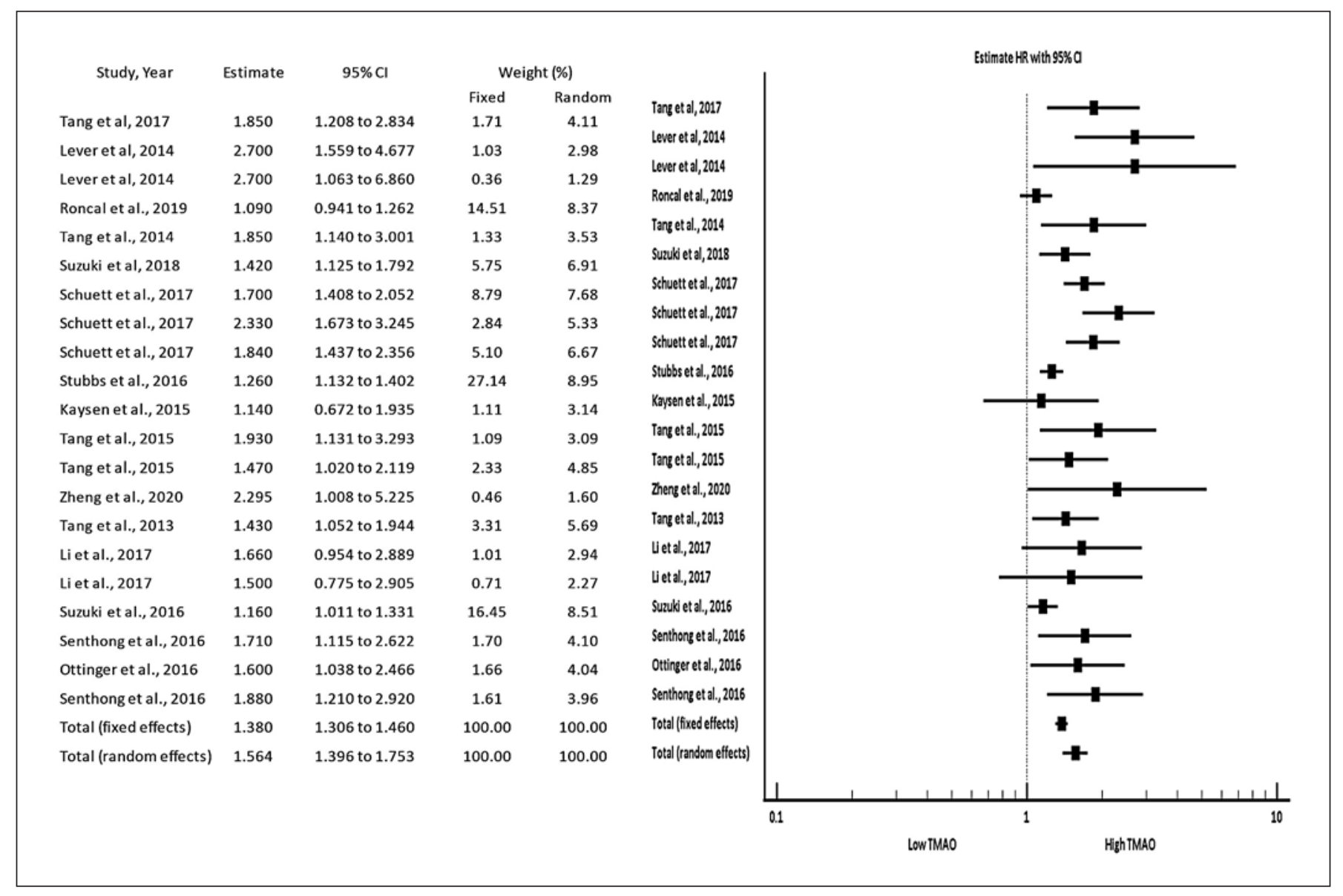

Fig. 2. Forest plot showing association of TMAO with all-cause mortality.

MeSH terms “TMAO OR Trimethylamine-N-oxide” combined with "Cardiovascular Disease OR Mortality OR Cardiovascular Mortality." The search algorithm for Pubmed was as follows (trimethylamine-N-oxide OR TMAO) AND (cardiovascular disease OR coronary heart disease OR stroke OR heart failure OR ischemic heart disease OR myocardial infarction OR acute coronary syndrome OR sudden cardiac arrest OR cardiovascular death OR mortality). A manual search was also conducted on the references of selected articles to retrieve additional eligible studies. Studies had selected if the free full text was available in English among the elderly age-group defined as 60 years and above as it varies in the different regions.

\section{Selection Criteria}

For the present systematic review and meta-analysis, studies had to satisfy the following inclusion criteria: (i) studies conducted upon humans; (ii) prospective cohort study design; (iii) baseline plasma or serum trimethylamine-N-oxide level values available; (iv) major cardiovascular events (including cardiovascular mortality, cardiovascular hospitalization, revascularization, myocardial infarction [MI], and stroke) or all-cause mortality were described as the end-points; and (v) HRs with their 95\% confidence intervals ( $95 \%$ CIs) were reported or could be estimated as for the associa- tion between baseline TMAO and adverse cardiovascular events and/or mortality. Studies were excluded if any of the ensuing was present: (i) duplicate publication; (ii) lack of data on TMAO plasma levels and their correlation with outcomes; (iii) the outcome of interest was not reported or was impossible to extract or calculate from the published results; and (iv) the number of included patients. We also excluded studies that were narrative reviews, abstracts, meeting proceedings, personal communications, case reports, or presented insufficient data for pooling. We included data with adjustments for confounding factors, such as age, sex, eGFR, or traditional CVD risk factors, wherever present. Two authors (G.C. and L.H.) independently examined the title and abstract of citations. The full texts of potentially selected studies were reviewed, and any disagreements were resolved by discussion.

\section{Data Extraction}

A data extraction sheet was predesigned wherein surname of the first author, year of publication, country of origin, sample size studied, follow-up period, sex proportion, mean age, baseline comorbidity, and selected biochemical parameters, disease outcome, serum TMAO, HRs (or ORs, RRs) with their corresponding 95\% CIs were extracted for each included study. The outcome assessed in the meta-analysis included risk of CVD, all-cause mor- 


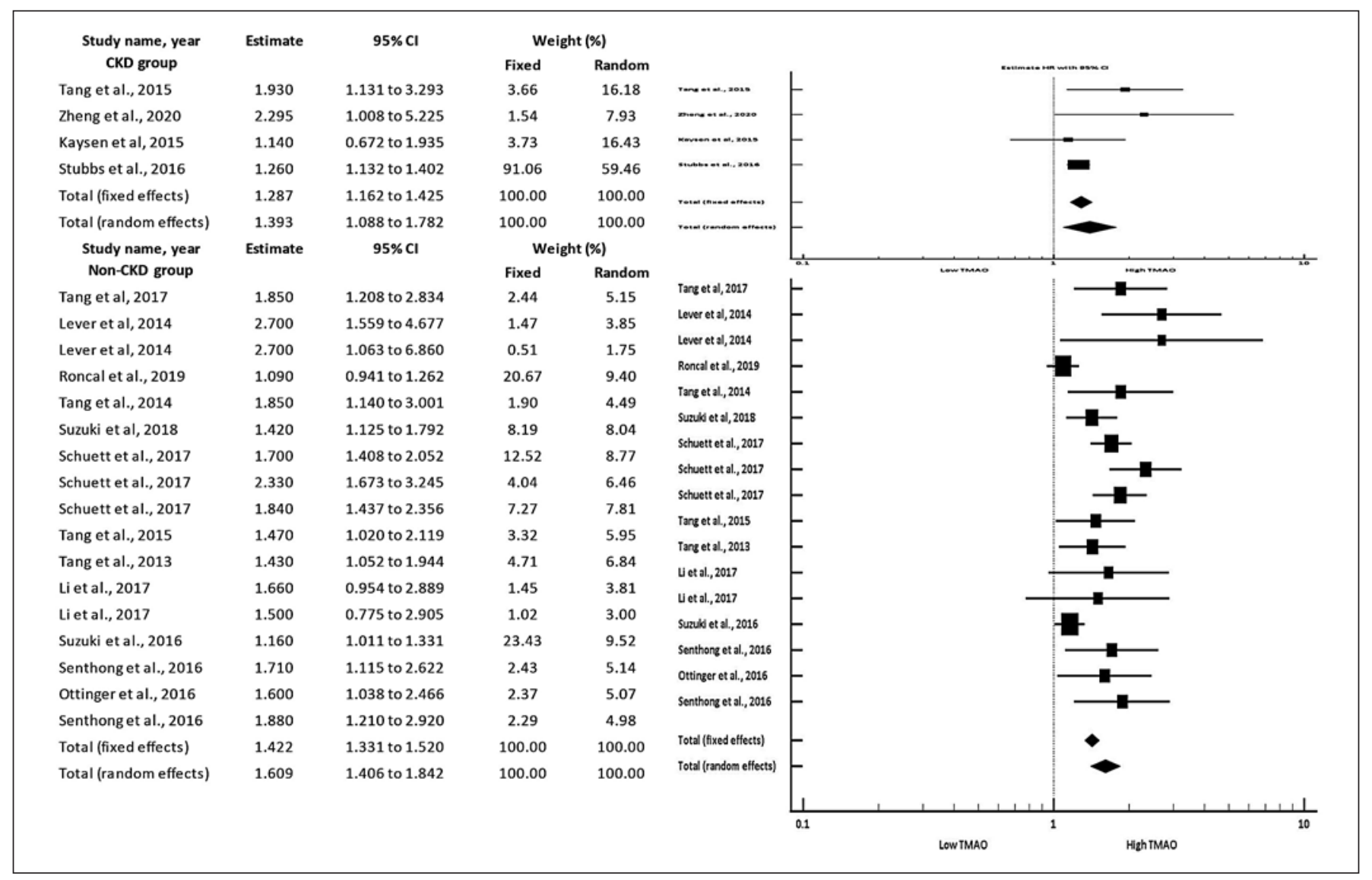

Fig. 3. Subgroup analysis for plasma TMAO levels and all-cause mortality in CKD versus non-CKD patients.

tality, and major adverse cardiac and cerebrovascular event (MACE), including congestive $\mathrm{HF}, \mathrm{MI}$, death due to cardiac causes, and stroke.

\section{Quality Assessment of Included Studies}

The quality of selected studies was assessed using the Newcastle-Ottawa Scale (NOS) based on study group selection, comparability, and ascertainment of exposure or outcome (Table 2).

\section{Data Analysis}

We used HRs, and the corresponding CIs were reported in individual studies to estimate the risk of cardiovascular outcome for the patients with the highest quartile compared with the lowest quartile TMAO levels, wherever reported. Other studies reported TMAO levels in variable units, including tertiles, per SD increasing or log-transformed. We log-transformed all HRs and derived the log-transformed SEs from reported CIs. Summary HRs and CIs from adjusted primary sources were abstracted. We assumed that the exposure variable (TMAO) is usually distributed, and the association with all-cause mortality and/or adverse cardiovascular events is log-linear. We used the generic inverse variance method of meta-analysis for log-transformed estimates. The Cochran's Q test was used to assess heterogeneity among studies and quantified using the $I^{2}$ static. A random-effects model was applied for calcu- lating pooled HRs and their CIs. A fixed-effect model was also used when heterogeneity was absent $\left(I^{2}=0 \%\right)$.

Subgroup analyses by comorbidity, including kidney disease, HF, and diabetes mellitus, were performed, indicating potential sources of heterogeneity. The assessment of effect was calculated with the random-effects model and expressed as HRs. The statistical significance was set at $p<0.05$ (2-tailed). Publication bias and selective reporting were examined by visual inspection of the asymmetry of the funnel plot, where the log SE of log HR was plotted against HR. Egger's and Begg's linear regression tests were conducted to determine statistical significance. All statistical analyses were performed using STATA software, version 14.1 (StataCorp LP, College Station, TX, USA), and MedCalc statistical software, version 20.0.4. A two-sided $p$ value $<0.05$ was considered significant.

\section{Results}

\section{Literature Search}

The initial search of the literature identified 1,292 articles from various databases, of which 198 full-text articles were retrieved for review, and 27 cohort studies were 


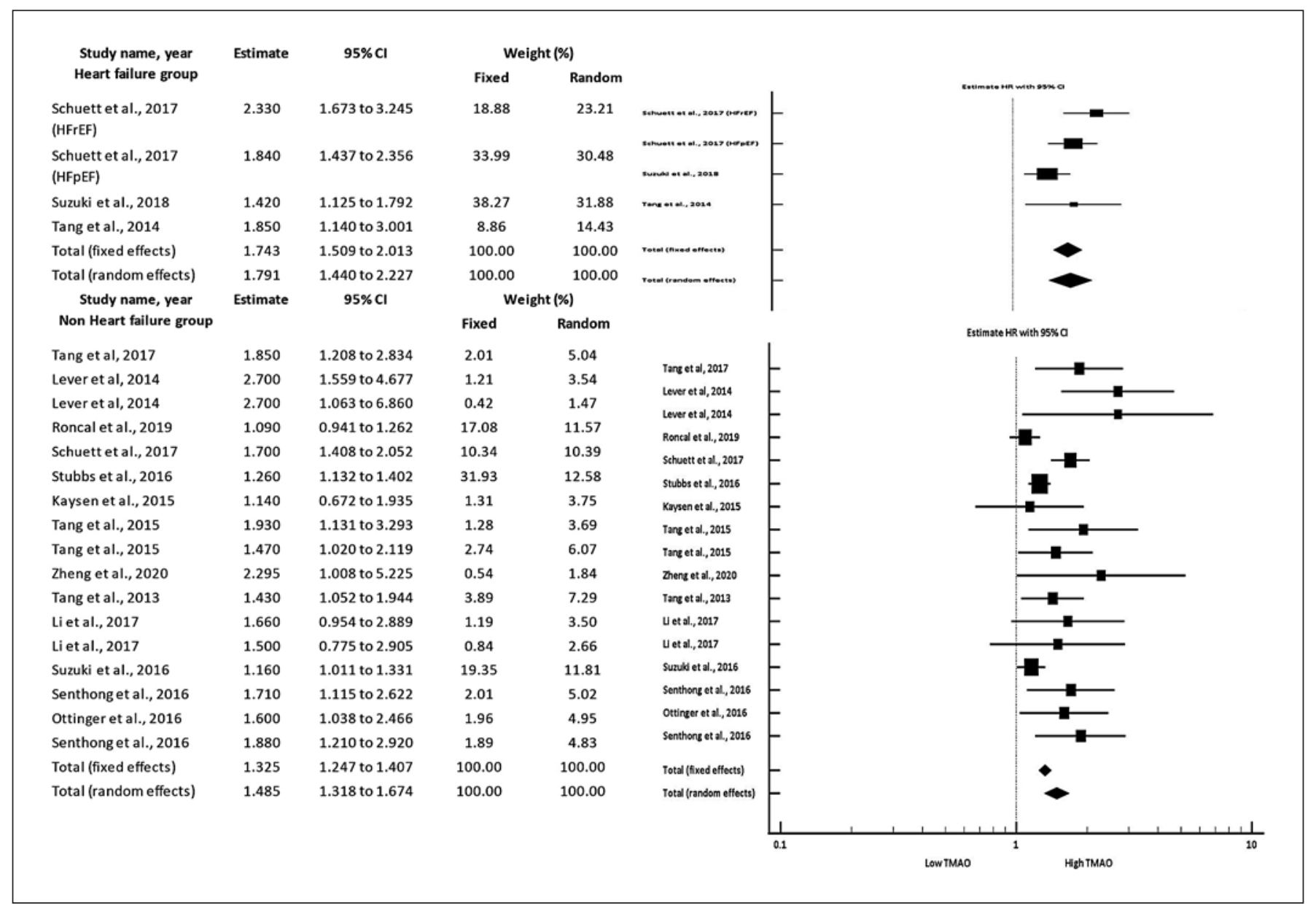

Fig. 4. Subgroup analysis for plasma TMAO levels and all-cause mortality in HF versus non-HF patients.

finally included in the meta-analysis (Fig. 1). Characteristics and baseline data of the included studies are provided in Table 1.

\section{Characteristics of Studies Included for Systematic Review}

The detailed characteristics of the 27 prospective studies published from inception (2013) to 2020 are shown in Table 1. Among these studies, thirteen were conducted in the USA $[3-5,11-20]$, while others were performed in UK [6, 21, 22], New Zealand [23], Canada [12, 24], Germany [25, 26], Sweden [27], Japan [10], Spain [28], China $[29,30]$, Switzerland [11, 31], and Austria [32]. The population included in the cohorts varied from 69 [29] to 4,007 [4, 17] participants, with 39,206 subjects included in the final meta-analysis. Follow-up durations of these studies ranged from $1.0[6,26,29]$ to 9.7 [25] years. Categories of patients included patients undergoing elective coronary angiography, patients with coronary artery disease, patients with stroke, patients with HF, patients with diabetes, patients with peripheral artery disease, and patients with chronic kidney disease (CKD). One study [31] that presented outcomes for CAP patients with or without comorbidity of coronary artery disease was treated as two cohorts.

Similarly, Li et al. [11] also presented data for two cohorts, while Schuett et al. [25] had three cohorts. The outcome reported from these studies was all-cause mortality and cardiovascular events, including MI, coronary revascularization, stroke, cardiovascular hospitalization, and mortality. The mean or median value of the TMAO levels are ranged from 1.74 [32] to $43 \mathrm{mmol} / \mathrm{L}$ [16] across the 27 prospective studies listed in Table 1 . The HRs of high TMAO measured as a continuous variable ranging from 1.01(CI, 0.984-1.04) [32] to 5.65 (CI, 1.91-16.74) [11] for adverse cardiovascular events (Table 2). Upon quality 


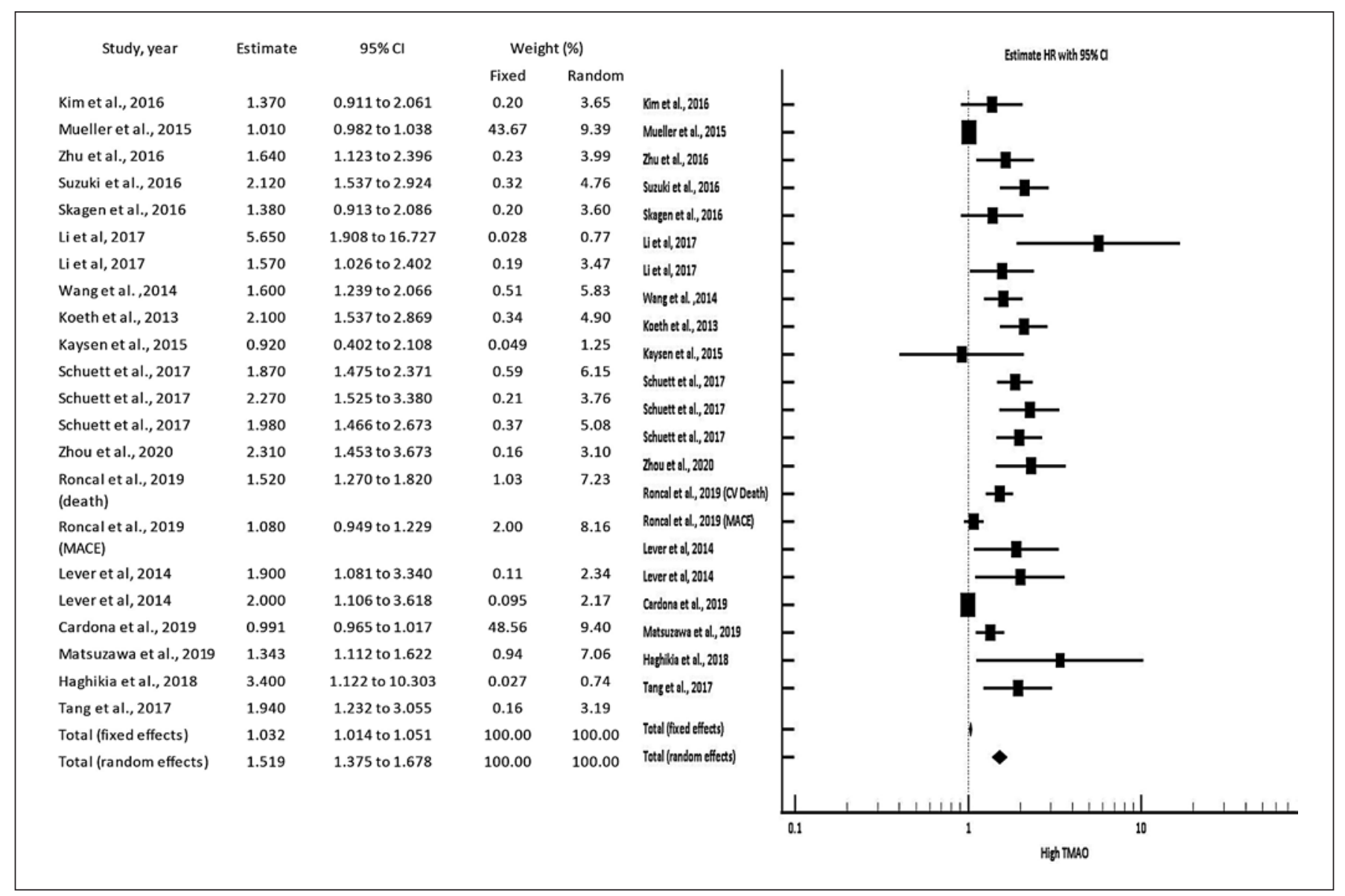

Fig. 5. Forest plot showing association of TMAO and adverse cardiovascular events and mortality.

rating using NOS, we found all included studies to be moderate-to-high quality, with individual study NOS scores ranging from 5 to 8 .

\section{Association between TMAO and All-Cause Mortality}

Upon meta-analysis of all studies, it was found that higher TMAO plasma levels were associated with greater risk of all-cause mortality (16 studies for 20 cohorts enrolling 23,331 subjects, HR: $1.38,95 \%$ CI: $1.306-1.460$, $p$ $<0.001, I^{2}=64.81 \%$; comparing patients with "high" vs. "low" TMAO, Fig. 2).

When the analysis for all-cause mortality was repeated stratifying the studies according to the presence or absence of CKD in the primary studies populations, a significant association between TMAO levels and all-cause mortality was found both in the CKD and non-CKD cohort (non-CKD: 13 studies for 17 cohorts enrolling 38,161 subjects, HR: 1.42, 95\% CI: 1.331-1.520, $p<0.001, I^{2}=$
67.86\%; CKD: 4 studies enrolling 1,045 subjects, HR: 1.393, 95\% CI: 1.088-1.782, $p=0.009, I^{2}=32.74 \%$ : Fig. 3 ).

We also stratified studies according to presence of $\mathrm{HF}$ and found that statistical significance for the association between all-cause mortality and TMAO levels persisted (non-HF: 15 studies for 18 cohorts enrolling 26,488 subjects, HR: 1.325 , 95\% CI: 1.247-1.407, $p<0.001, I^{2}=$ 58.76\%; HF: 3 studies enrolling 5,940 subjects, HR: 1.791, 95\% CI: $1.440-2.227, p=<0.001, I^{2}=51.86 \%$ : Fig. 4 ).

\section{Association between TMAO and Adverse Cardio and Cerebrovascular Events}

In patients with high TMAO plasma levels, the incidence of MACE was significantly higher compared with patients with low TMAO levels (seventeen studies for 22 cohorts enrolling 31,857 subjects, HR: 1.032, 95\% CI: $1.014-1.051, p=0.001, I^{2}=89.52 \%$; Fig. 5). We repeated the analysis for cardiovascular mortality/adverse events
48

Cardiorenal Med 2022;12:39-54 DOI: $10.1159 / 000520910$
Chen/He/Dou/Liu 


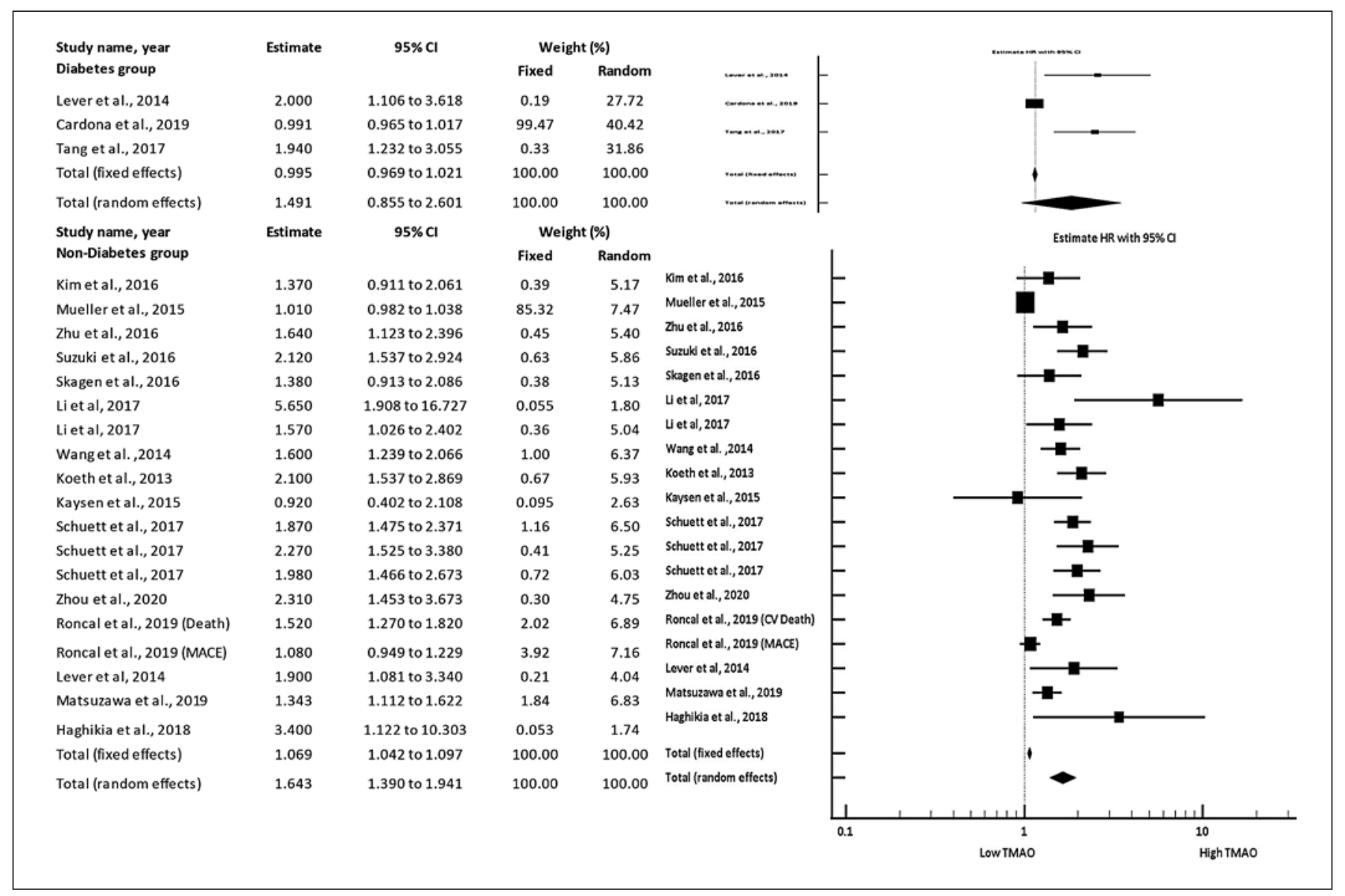

Fig. 6. Subgroup analysis for plasma TMAO levels and adverse cardiovascular mortality/events in diabetes versus nondiabetic patients.

by stratifying the studies according to the presence or absence of diabetes in the primary study populations. We found a significant association between TMAO levels and adverse CV events/mortality nondiabetes group, whereas in the diabetes group, it was not significant (nondiabetes: 15 studies for 18 cohorts enrolling 34,315 subjects, HR: $1.069,95 \%$ CI: $1.042-1.097, p<0.001, I^{2}=89.51 \%$; diabetes: 3 studies enrolling 4,891 subjects, HR: $1.491,95 \%$ CI: $0.855-2.601, p=0.159, I^{2}=85.43 \%$ : Fig. 6$)$.

\section{Study Quality Assessment and Heterogeneity}

The NOS was used to assess the quality of the selected studies. Quality assessment helps to identify heterogeneity within studies. Heterogeneity assessment, in turn, informs if the observed differences in results are a result of chance. Upon quality assessment, we found that all included studies fell into the categories "good" or "fair" (Table 3), allowing us to identify two subgroups ("good" and "fair") and perform a subgroup analysis of our primary outcome. Subgroup analysis also showed that high TMAO plasma levels were associated with higher all-cause mortality in both subgroups. Thus, the observed heterogeneity resulted due to the "fair quality" study group. It may also be explained by the variable population size of the studies included in the meta-analysis. Funnel plots and Egger's test did not show any publication bias for the analyses performed (Fig. 7, 8).

\section{Discussion}

The present meta-analysis of pooled data from 27 prospective cohort studies found that higher circulating TMAO at baseline was independently associated with 1.38 times increased risk of all-cause mortality and 1.032 times increment of adverse cardiovascular events among 


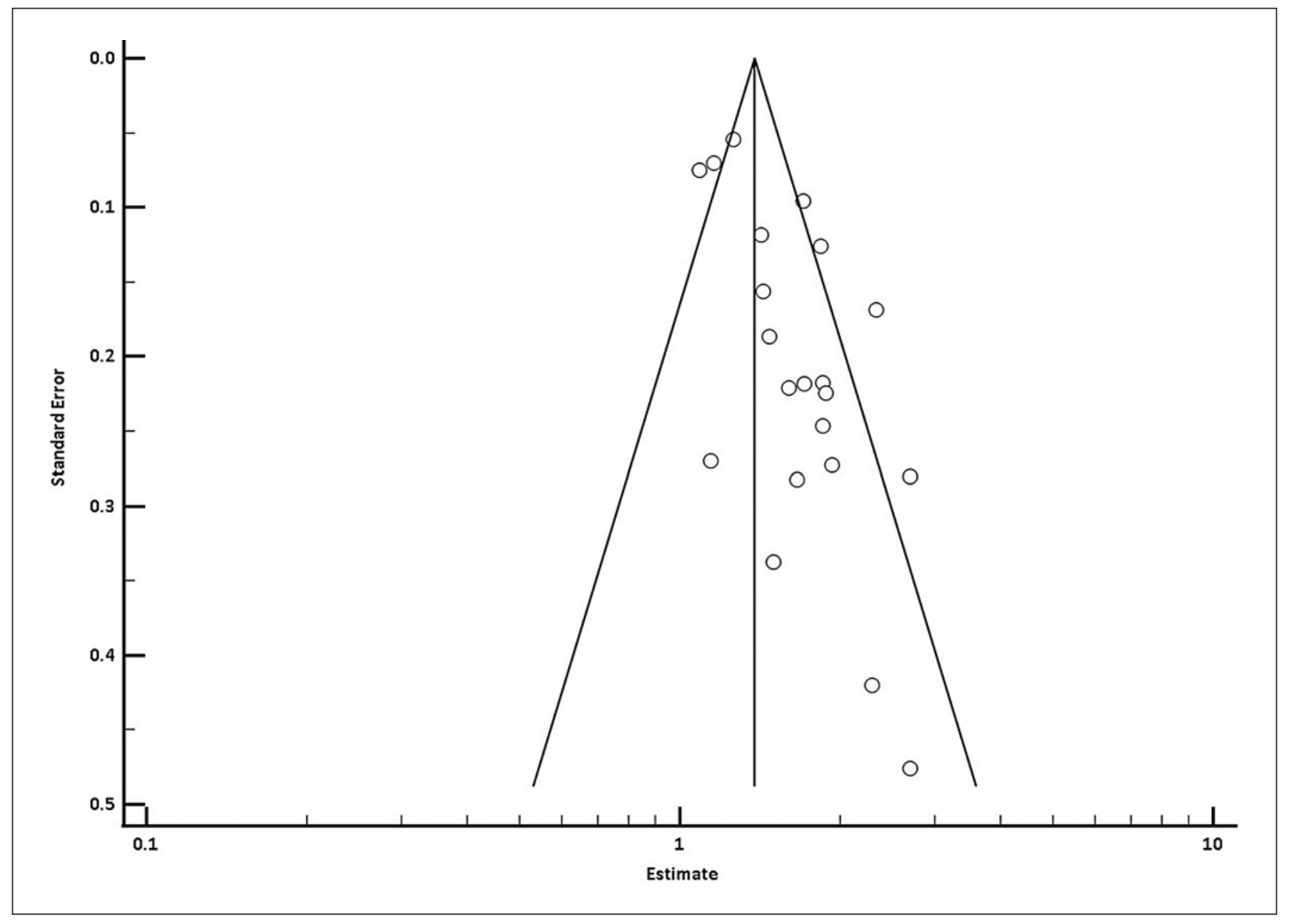

Fig. 7. Funnel plot of included studies for all-cause mortality.

the elderly age-group. The included studies were significantly heterogeneous. However, subsequent subgroup analysis using $\mathrm{CKD}, \mathrm{HF}$, and diabetes as subgroups also yielded consistent results regarding high TMAO levels with all-cause mortality and adverse cardiovascular outcomes. These results suggest that participants with higher baseline TMAO were at higher risk of future cardiovascular events and mortality due to any cause. Further extensive studies are needed to determine whether circulating TMAO can be seen as a potential biomarker for future mortality and cardiovascular risk. In addition, we found a lack of studies from many developing countries such as India, where cardiovascular risk factors are highly prevalent. It would be interesting to study the TMAO profile of such populations and its usefulness for predicting future cardiovascular events and mortality.
Most of the independent studies and reviews conducted in the past on associations of circulating TMAO have reported a positive association with future mortality and adverse cardiovascular outcomes, commonly referred to as MACE, a finding consistent with ours. The elevated concentrations of TMAO level were associated with a pooled RR of 1.62 (95\% CI, 1.45, 1.80; $I^{2}=23.5 \%$ ) for MACE compared with low TMAO levels; elevated TMAO level concentrations were associated with a pooled RR of 1.63 (95\% CI, 1.36, 1.95) for all-cause mortality [33]. Few studies that did not find this association attributed it to low TMAO concentrations, where it is cardioprotective [34].

Overall, the studies on associations between TMAO and CVD reported inconsistent results. We found a positive association of TMAO with future mortality and cardiovascular risk among patients with $\mathrm{CKD}, \mathrm{HF}$, and dia- 


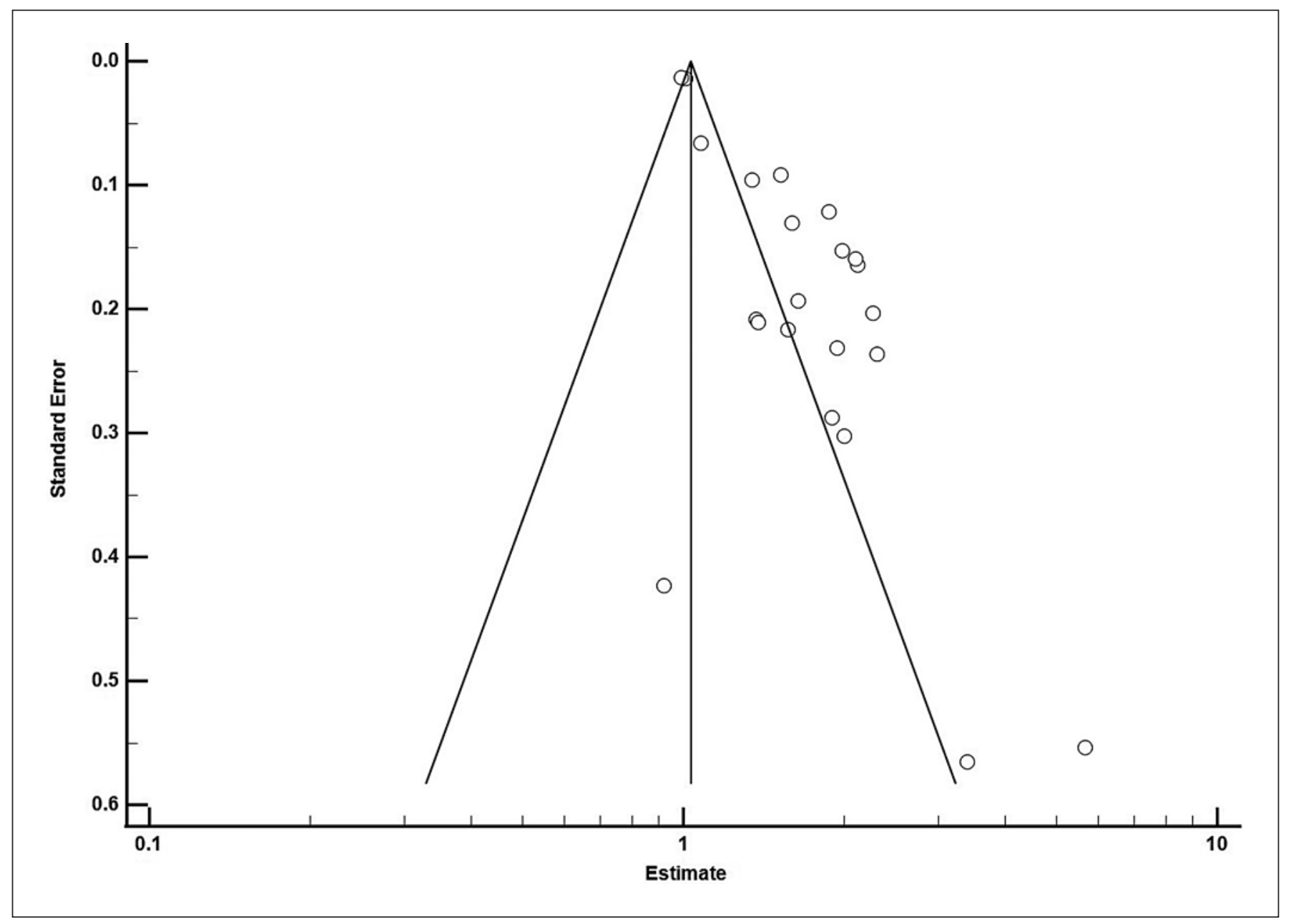

Fig. 8. Funnel plot of included studies for adverse cardiovascular events/mortality.

betes in subgroup analyses. This association has been explained earlier, wherein increased TMAO is associated with the consumption of foods such as eggs and fish. Several pathways involving precursors of TMAO have been proposed linking the elevated levels of TMAO to diet. For instance, Koeth et al. [3] showed that dietary supplementation of mice with choline or L-carnitine increased TMAO levels and enhanced the development of atherosclerosis. TMAO promotes insulin resistance and adipose inflammation in mouse models and is associated with an increased risk of developing type 2 diabetes in humans.

Additionally, other mechanisms such as a role in foam cell formations have also been suggested, contributing to atherogenesis and consequent cardiovascular sequelae. However, few suggested an increase in precursors of TMAO such as betaine, a metabolite of choline [2], and showed dietary betaine administration induced produc- tion of TMAO in animals [13]. Others suggested the role of precursors like L-carnitine in red meat that can be transformed to gamma-butyrobetaine by gut bacteria prior to TMA and TMAO [35]. Nevertheless, all have been shown to follow a similar pathway for increasing the plasma TMAO concentration and suggesting the possible role of TMAO as a mediator rather than a marker in the pathway.

Few studies found that relations of elevated TMAO level and its precursors with MACE and all-cause death were independent of predictable risk factors, such as kidney dysfunction, diabetes mellitus, and obesity, similar to our subgroup analysis [33]. Moreover, the selected studies included in this meta-analysis had a similarly aged population, implying that chronic diseases lose their impact on the risk of mortality in the elderly age-group since older adults already survived the risk when younger. 
Table 3. Quality assessment of included studies using NOS

\begin{tabular}{|c|c|c|c|c|c|}
\hline S. No. & Author & Selection & Comparability & Outcome & Total \\
\hline 1 & Tang et al. [4] & 4 & 2 & 2 & 8 \\
\hline 2 & Lever et al. [23] & 3 & 1 & 1 & 5 \\
\hline 3 & Skagen et al. [27] & 4 & 1 & 2 & 7 \\
\hline 4 & Suzuki et al. [6] & 3 & 1 & 1 & 5 \\
\hline 5 & Matsuzawa et al. [10] & 3 & 1 & 2 & 6 \\
\hline 6 & Schuett et al. [25] & 3 & 1 & 1 & 5 \\
\hline 7 & Suzuki et al. [21] & 3 & 1 & 1 & 5 \\
\hline 8 & Kim et al. [24] & 2 & 1 & 2 & 5 \\
\hline 9 & Li et al. [11] & 4 & 1 & 2 & 7 \\
\hline 10 & Zheng et al. [29] & 3 & 1 & 1 & 5 \\
\hline 11 & Kaysen et al. [16] & 2 & 1 & 2 & 5 \\
\hline 12 & Tang et al. [15] & 4 & 2 & 2 & 8 \\
\hline 13 & Ottiger et al. [31] & 3 & 1 & 1 & 5 \\
\hline 14 & Zhou et al. [30] & 2 & 1 & 2 & 5 \\
\hline 15 & Roncal et al. [28] & 3 & 1 & 1 & 5 \\
\hline 16 & Cardona et al. [12] & 2 & 1 & 2 & 5 \\
\hline 17 & Suzuki et al. [6] & 3 & 1 & 2 & 6 \\
\hline 18 & Haghikia et al. [26] & 3 & 1 & 1 & 5 \\
\hline 19 & Koeth et al. [3] & 3 & 1 & 2 & 6 \\
\hline 20 & Wang et al. [2] & 4 & 2 & 2 & 8 \\
\hline 21 & Tang et al. [14] & 4 & 2 & 2 & 8 \\
\hline 22 & Mueller et al. [32] & 3 & 1 & 1 & 5 \\
\hline 23 & Senthong et al. [5] & 3 & 1 & 2 & 6 \\
\hline 24 & Senthong et al. [5] & 3 & 1 & 2 & 6 \\
\hline 25 & Stubbs et al. [19] & 3 & 1 & 2 & 6 \\
\hline 26 & Zhu et al. [17] & 2 & 1 & 2 & 5 \\
\hline 27 & Tang et al. [20] & 3 & 2 & 2 & 7 \\
\hline
\end{tabular}

\section{Strength and Limitations}

The strengths of this study are many. First, we included only prospective original studies to ascertain that reverse causation would be minimized in analysis. Second, we adjusted rates from the studies that addressed the influence of several potential confounders and risk factors for CVDs. Moreover, the definition of elevated TMAO levels was different across individual studies, as were the methods of assessment. We, therefore, used the log-transformed values to harmonize the differences. Third, the included studies represented populations from the USA and European countries, China, and Japan, which primarily have not been included in previous analyses, therefore enabling us to generalize our findings to all parts of the world, partly. However, a few limitations remained. First, none of the included studies had data from the general population. In addition, the included studies represented clinical cohorts that included patients with preexisting cardiovascular risk. Therefore, this analysis limits the generalization of our findings to a low-risk population. Second, despite using cohort studies, we could not determine the causal association between the high TMAO levels and adverse cardiovascular events causing the adverse effects or vice versa. Third, a minimum possibility of residual confounding attributed to unmeasured factors also remained, although we included hazard rates from the adjusted models reported in the studies. Finally, the adverse outcomes related to high TMAO levels are affected by sex or race/ethnicity are not available from the selected studies.

\section{Conclusion}

In this meta-analysis of patients in the elderly agegroup, high TMAO was positively associated with allcause mortality (HR: 1.38 [95\% CI: 1.306-1.460]) and adverse cardiovascular events (HR: 1.032 [95\% CI: 1.0141.051]). However, owing to high heterogeneity in the studies, we recommend larger cross-sectional studies need to be conducted in the general population better to understand the risks of mortality with high TMAO levels. 


\section{Statement of Ethics}

Not required, as it is a systematic review and meta-analysis paper.

\section{Conflict of Interest Statement}

The authors declare that they have no competing interests.

\section{Funding Sources}

This project has been funded by Nanchong City school strategic cooperation science and technology project (Metabonomics of coronary heart disease and its correlation with intestinal flora in Northeast Sichuan region, 18SXHZ0436) received for this study.

\section{Author Contributions}

G.C. has made substantial contributions to conception and design of the study; L.H. and X.D. searched literature, extracted data from the collected literature, and analyzed the data; G.C. and L. wrote the manuscript; T.L. revised the manuscript. All authors approved the final version of the manuscript.

\section{Data Availability Statement}

All data generated and analyzed in the study are available from the corresponding author upon reasonable request.

\section{References}

1 Devereaux PJ, Sessler DI. Cardiac complications in patients undergoing major noncardiac surgery. N Engl J Med. 2015;373(23): 2258-69.

2 Wang Z, Klipfell E, Bennett BJ, Koeth R, Levison BS, Dugar B, et al. Gut flora metabolism of phosphatidylcholine promotes cardiovascular disease. Nature. 2011;472:57-63.

3 Koeth RA, Wang Z, Levison BS, Buffa JA, Org E, Sheehy BT, et al. Intestinal microbiota metabolism of 1 carnitine, a nutrient in red meat, promotes atherosclerosis. Nat Med. 2013;19: 576-85.

4 Tang WH, Wang Z, Levison BS, Koeth RA, Britt EB, Fu X, et al. Intestinal microbial metabolism of phosphatidylcholine and cardiovascular risk. N Engl J Med. 2013;368:157584.

5 Senthong V, Wang Z, Li XS, Fan Y, Wu Y, Wilson Tang $\mathrm{WH}$, et al. Intestinal microbiota generated metabolite trimethylamine $\mathrm{N}$ oxide and 5 year mortality risk in stable coronary artery disease: the contributory role of intestinal microbiota in a COURAGE like patient cohort. J Am Heart Assoc. 2016;5:e002816002823.

6 Suzuki T, Heaney LM, Bhandari SS, Jones DJ, $\mathrm{Ng}$ LL. Trimethylamine $\mathrm{N}$-oxide and prognosis in acute heart failure. Heart. 2016;102: 841-8.

7 Farhangi MA. Gut microbiota-dependent trimethylamine $\mathrm{N}$-oxide and all-cause mortality: findings from an updated systematic review and meta-analysis. Nutrition. 2020;78: 110856.

8 United Nations, Department of Economic and Social Affairs, Population Division. World population ageing 2019: Highlights (ST/ESA/SER.A/430); 2019.
9 Rodgers JL, Jones J, Bolleddu SI, Vanthenapalli S, Rodgers LE, Shah K, et al. Cardiovascular risks associated with gender and aging. J Cardiovasc Dev Dis. 2019 Apr 27;6(2):19.

10 Matsuzawa Y, Nakahashi H, Konishi M, Sato R, Kawashima C, Kikuchi S, et al. Microbiotaderived Trimethylamine $\mathrm{N}$-oxide predicts cardiovascular risk after STEMI. Sci Rep. 2019;9:11647.

11 Li XS, Obeid S, Klingenberg R, Gencer B, Mach F, Räber L, et al. Gut microbiota-dependent trimethylamine $\mathrm{N}$-oxide in acute coronary syndromes: a prognostic marker for incident cardiovascular events beyond traditional risk factors. Eur Heart J. 2017;38: 814-24.

12 Cardona A, O’Brien A, Bernier MC, Somogyi A, Wysocki VH, Smart S, et al. Trimethylamine $\mathrm{N}$-oxide and incident atherosclerotic events in high-risk individuals with diabetes: an ACCORD trial post hoc analysis. BMJ Open Diabetes Res Care. 2019;7(1):e000718.

13 Wang Z, Tang WH, Buffa JA, Fu X, Britt EB, Koeth RA, et al. Prognostic value of choline and betaine depends on intestinal microbiota-generated metabolite trimethylamine- $\mathrm{N}$ oxide. Eur Heart J. 2014;35:904-10.

14 Tang WH, Wang Z, Fan Y, Levison B, Hazen JE, Donahue LM, et al. Prognostic value of elevated levels of intestinal microbe generated metabolite trimethylamine $\mathrm{N}$ oxide in patients with heart failure: refining the gut hypothesis. J Am Coll Cardiol. 2014;64:190814.

15 Tang WH, Wang Z, Kennedy DJ, Wu Y, Buffa JA, Agatisa-Boyle B, et al. Gut microbiotadependent trimethylamine $\mathrm{N}$-oxide (TMAO) pathway contributes to both development of renal insufficiency and mortality risk in chronic kidney disease. Circ Res. 2015;116: $448-55$.
16 Kaysen GA, Johansen KL, Chertow GM, Dalrymple LS, Kornak J, Grimes B, et al. Associations of trimethylamine $\mathrm{N}$-oxide with nutritional and inflammatory biomarkers and cardiovascular outcomes in patients new to dialysis. J Ren Nutr. 2015;25:351-6.

17 Zhu W, Gregory JC, Org E, Buffa JA, Gupta $\mathrm{N}$, Wang $\mathrm{Z}$, et al. Gut microbial metabolite TMAO enhances platelet hyperreactivity and thrombosis risk. Cell. 2016;165:111-24.

18 Senthong V, Wang Z, Wu Y, Tang WH, Hazen S. Elevated plasma trimethylamine $\mathrm{N}$ oxide is associated with poor prognosis in patients with peripheral artery disease. J Am Coll Cardiol. 2015;65:2076-9.

19 Stubbs JR, House JA, Ocque AJ, Zhang S, Johnson C, Kimber C, et al. Serum trimethylamine $\mathrm{N}$ oxide is elevated in $\mathrm{CKD}$ and correlates with coronary atherosclerosis burden. J Am Soc Nephrol. 2016;27:305-13.

20 Tang WH, Wang Z, Li XS, Fan Y, Li DS, Wu $\mathrm{Y}$, et al. Increased trimethylamine $\mathrm{N}$ oxide portends high mortality risk independent of glycemic control in patients with type 2 diabetes mellitus. Clin Chem. 2017;63:297-06.

21 Suzuki T, Yazaki Y, Voors AA, Jones DJL, Chan DCS, Anker SD, et al. Association with outcomes and response to treatment of trimethylamine $\mathrm{N}$-oxide in heart failure: results from BIOSTAT-CHF. Eur J Heart Fail. 2019; 21(7):877-86.

22 Suzuki T, Heaney LM, Jones DJ, Ng LL. Trimethylamine $\mathrm{N}$-oxide and risk stratification after acute myocardial infarction. Clin Chem. 2017;63(1):420-8.

23 Lever M, George PM, Slow S, ang M, Muirhead N, Barrett B, et al. Betaine and trimethylamine $\mathrm{N}$ oxide as predictors of cardiovascular outcomes show different patterns in diabetes mellitus: an observational study. PLoS One. 2014;9:e114969. 
24 Kim RB, Morse BL, Djurdjev O, Tang M, Muirhead N, Barrett B, et al. Advanced chronic kidney disease populations have elevated trimethylamine $\mathrm{N}$ oxide levels associated with increased cardiovascular events. Kidney Int. 2016;89:1144-52.

25 Schuett K, Kleber ME, Scharnagl H, Lorkowski S, März W, Niessner A, et al. Trimethylamine- $\mathrm{N}$-oxide and heart failure with reduced versus preserved ejection fraction. J Am Coll Cardiol. 2017;70(25):3202-4.

26 Haghikia A, Li XS, Liman TG, Bledau N, Schmidt D, Zimmermann F, et al. Gut microbiota-dependent trimethylamine $\mathrm{N}$-oxide predicts risk of cardiovascular events in patients with stroke and is related to proinflammatory monocytes. Arterioscler Thromb Vasc Biol. 2018;38(9):2225-35.

27 Skagen K, Troseid M, Ueland T, Holm S, Abbas A, Gregersen I, et al. The carnitine butyrobetaine trimethylamine $\mathrm{N}$ oxide pathway and its association with cardiovascular mortality in patients with carotid atherosclerosis. Atherosclerosis. 2016;247:64-9.
28 Roncal C, Martínez-Aguilar E, Orbe J, Ravassa S, Fernandez-Montero A, Saenz-Pipaon $\mathrm{G}$, et al. Trimethylamine-N-oxide (TMAO) predicts cardiovascular mortality in peripheral artery disease. Sci Rep. 2019;9(1):15580.

29 Zheng Y, Tang Z, You L, Wu Y, Liu J, Xue J. Trimethylamine- $\mathrm{N}$-oxide is an independent risk factor for hospitalization events in patients receiving maintenance hemodialysis. Ren Fail. 2020;42(1):580-6.

30 Zhou X, Jin M, Liu L, Yu Z, Lu X, Zhang H. Trimethylamine $\mathrm{N}$-oxide and cardiovascular outcomes in patients with chronic heart failure after myocardial infarction. ESC Heart Fail. 2020;7(1):188-93.

31 Ottiger M, Nickler M, Steuer C, Odermatt J, Huber A, Christ-Crain M, et al. Trimethylamine $\mathrm{N}$ oxide (TMAO) predicts fatal outcomes in community acquired pneumonia patients without evident coronary artery disease. Eur J Intern Med. 2016;36:67-73.
32 Mueller DM, Allenspach M, Othman A, Saely $\mathrm{CH}$, Muendlein A, Vonbank A, et al. Plasma levels of trimethylamine- $\mathrm{N}$-oxide are confounded by impaired kidney function and poor metabolic control. Atherosclerosis. 2015;243:638-44.

33 Heianza Y, Ma W, Manson JE, Rexrode KM, Qi L. Gut microbiota metabolites and risk of major adverse cardiovascular disease events and death: a systematic review and meta analysis of prospective studies. J Am Heart Assoc. 2017;6:e004947.

34 Collins HL, Drazul-Schrader D, Sulpizio AC, Koster PD, Williamson Y, Adelman SJ, et al. l-carnitine intake and high trimethylamine $\mathrm{N}$-oxide plasma levels correlate with low aortic lesions in ApoE-/- transgenic mice expressing CETP. Atherosclerosis. 2016;244: 29-37.

35 Bennett BJ, de Aguiar Vallim TQ, Wang Z, Shih DM, Meng Y, Gregory J, et al. Trimethylamine $\mathrm{N}$ oxide, a metabolite associated with atherosclerosis, exhibits complex genetic and dietary regulation. Cell Metab. 2013;17:4960. 